\title{
Relação entre Transtornos Mentais Comuns e a ingestão dietética de universitários da área da saúde
}

\author{
Association between common mental disorders and dietary intake \\ among university students doing health-related courses
}

\begin{abstract}
Antoniel Rodrigues Sousa (https://orcid.org/0000-0001-8636-2691) ${ }^{1}$
Dayanna Magalhães dos Reis (https://orcid.org/0000-0002-0213-0824) ${ }^{1}$

Thaís Meirelles de Vasconcelos (https://orcid.org/0000-0003-0108-8991) ${ }^{1}$

Ana Paula Vasconcellos Abdon (https://orcid.org/0000-0002-1597-1817) ${ }^{2}$

Soraia Pinheiro Machado (https://orcid.org/0000-0002-3918-4738) ${ }^{1}$

Ilana Nogueira Bezerra (https://orcid.org/0000-0002-2072-0123) ${ }^{1}$
\end{abstract}

${ }^{1}$ Centro de Ciências da Saúde, Universidade Estadual do Ceará. Av. Dr. Silas Munguba 1.700 Campus do Itaperi, 60714 903. Fortaleza CE Brasil. antonielr32@gmail.com

${ }^{2}$ Universidade de Fortaleza. Fortaleza CE Brasil.

\begin{abstract}
The objective of this study was to determine the association between common mental disorders (CMD) and dietary intake among Brazilian undergraduate students doing health-related courses. We interviewed 432 students enrolled at a public university in Fortaleza, the capital of the State of Ceará, between April and December 2018. The students were screened for CMD using the Self-Reporting Questionnaire (SRQ-20). Food consumption and physical activity were assessed using a 24-hour dietary recall and the International Physical Activity Questionnaire short form (IPAQ-SF), respectively. We used linear regression to measure the association between energy and nutrient intake and positive screening for CMD. The models were adjusted for age, gender and physical activity. Overall prevalence of CMD was $44.5 \%$ and the rate was higher in women than in men. Individuals who screened positive for CMD practiced less physical activity and reported higher mean intake of added sugar and saturated fat and lower mean fiber intake, regardless of age, gender, and level of physical activity. Our findings show that the prevalence of CMD among undergraduate students doing health-related courses was high and that the presence of potential CMD was associated with higher intake of added sugar and saturated fats and lower fiber intake.
\end{abstract}

Key words Mental disorders, Students, Food consumption, Lifestyle
Resumo O objetivo foi identificar a relação entre Transtorno Mental Comum (TMC) e a ingestão dietética de universitários da área saúde. Foram entrevistados 432 estudantes, regularmente matriculados em um dos cursos da área da saúde em uma universidade pública, no município de Fortaleza, Ceará, entre abril e dezembro de 2018. Utilizou-se o Self Reporting Questionnaire-20 (SRQ20) para rastreio de TMC. O consumo alimentar foi avaliado por meio do Recordatório de 24 horas e, para a avaliação da atividade física, utilizou-se o International Physical Activity Questionnaire (IPAQ), versão curta. Modelos de regressão linear foram utilizados para avaliar a relação entre ingestão de energia e nutrientes, e rastreamento positivo para TMC. Os modelos foram ajustados por idade, sexo e atividade física. A prevalência de TMC foi de 44,5\%, sendo maior nas mulheres. Os indivíduos com rastreamento positivo para TMC apresentaram menor frequência de atividade fisica e maior média de ingestão de açúcar de adição, gordura saturada e menor média de ingestão de sódio e fibras, independente da idade, sexo e atividade fisica. O presente trabalho demonstrou altas prevalências de TMC entre os universitários, estando associada com maior ingestão de açúcar de adição e gordura saturada e menor ingestão de fibras.

Palavras-chave Transtorno mental, Estudantes, Consumo alimentar, Estilo de vida 


\section{Introdução}

Estudantes universitários se deparam com desafios que abrangem não só a formação acadêmica, como também circunstâncias sociais, afetivas e psicológicas, tornando-os propensos ao desenvolvimento de transtornos mentais ${ }^{1}$. Os Transtornos Mentais Comuns (TMC) caracterizamse pela dificuldade de concentração, de tomada de decisões, insônia, irritabilidade, fadiga, assim como queixas somáticas, como por exemplo, cefaleia, falta de apetite, tremores, má digestão, dentre outros ${ }^{2}$, podendo influenciar direta e negativamente o desempenho acadêmico e a qualidade de vida ${ }^{3}$.

Estudos nacionais e internacionais têm sinalizado elevadas prevalências de TMC podendo variar de e $28,8 \%$ a $44,7 \%$ entre os estudantes universitários ${ }^{4}$. Estudos realizados em universidades brasileiras evidenciaram prevalências de TMC variando de $31,5 \%$ a $44,9 \%$ entre os universitários da área da saúde ${ }^{5,6}$. Dentre as principais situações estressoras frente às demandas acadêmicas, encontram-se relatos de poucas horas de sono, sentir-se desesperado e desamparado, dificuldade de fazer novos amigos, além de timidez, medo excessivo e depressão? .

O consumo alimentar de universitários é rico em energia, açúcares de adição e sódio, favorecido pelo frequente consumo de lanches comercializados em cantinas, como fast foods, batatas fritas, bolos, tortas e bebidas açucaradas, enquanto a ingestão de frutas e legumes estão abaixo das recomendações ${ }^{8,9}$. A escolha por esses tipos de alimentos práticos e com baixa ou nenhuma contribuição de nutrientes essenciais para manutençãoda saúde está relacionada, muitas vezes, pelo distanciamento do contexto familiar, como também pela falta de tempo para autocuidado ${ }^{10}$.

Evidências sugerem que o consumo alimentar adequado pode exercer influência positiva na proteção ao desenvolvimento ou agravamento dos sintomas para $\mathrm{TMC}^{11}$. Considerando as exigências físicas, psicológicas e sociais ocasionadas pelo ingresso na universidade e a possível relação entre fatores dietéticos e transtornos mentais o presente estudo tem por objetivo identificar a relação entre o desenvolvimento do TMC e a ingestão de energia, açúcar de adição e sódio de universitários da área da saúde.

\section{Metodologia}

Trata-se de um estudo transversal, realizado com universitários de ambos os sexos, matriculados em uma universidade pública, no município de Fortaleza, estado do Ceará. A pesquisa faz parte de um estudo maior intitulado "Promoção de saúde na população jovem: qual o papel da Universidade?"

O universo amostral corresponde aos estudantes dos cursos do Centro de Ciências da Saúde (CCS). A definição do tamanho da amostra deu-se a partir da fórmula para população finita desenvolvida por $\operatorname{Cochran}^{12} \mathrm{e}$ a seleção dos participantes do estudo foi por conveniência. Para o cálculo do tamanho amostral de 381 alunos considerou-se o cálculo com a forma finita, utilizando a prevalência de excesso de peso de 50\% para maximizar o tamanho da amostra e por estar próximo da prevalência de excesso de peso no Brasil ${ }^{13}$, intervalo de confiança de $95 \%$, erro máximo de $5 \%$, considerando taxa de não resposta de $20 \%$ e universo amostral de 1.490 alunos matriculados no primeiro semestre de 2018. O número de discentes foi proporcional a quantidade de matriculados em cada curso.

Não foram considerados elegíveis estudantes que não estavam matriculados no período de coleta dos dados e aqueles que possuíam idade inferior a 18 anos. Nesta análise foram incluídos apenas os indivíduos que completaram as informações para todos os dados coletados.

A coleta de dados foi realizada de abril a dezembro de 2018 por equipe treinada, composta por psicóloga, nutricionistas e acadêmicos de nutrição. Aplicou-se o questionário que apresentava questões sobre características sociodemográficas (sexo, idade, raça/cor da pele, renda familiar, estado civil, curso matriculado e semestre em curso) e de estilo de vida (prática de atividade física, consumo de bebida alcoólica, uso de cigarro e excesso de peso). Para fins de análise algumas variáveis foram categorizadas, tais como: raça (branca e outras), idade ( $\leq 21$ anos e $>21$ anos), semestre em curso (início $\leq 4^{\circ}$ e final $\geq 5^{\circ}$ ), renda familiar ( $\leq 3$ salários $>3$ salários) e estado civil (solteiro e outros).

Para avaliação da atividade física utilizou-se o International Physical Activity Questionnaire (IPAQ), versão curta $^{14}$. O instrumento é composto por perguntas direcionadas às atividades realizadas na última semana anterior à aplicação do questionário. Os alunos foram avaliados e posteriormente classificados, segundo o Centro Coordenador do IPAQ no Brasil ${ }^{15} \mathrm{em}$ : Muito 
ativo, indivíduo que cumpriu as recomendações de atividade vigorosa por cinco ou mais dias na semana e durante 30 ou mais minutos por sessão. Ou de atividade vigorosa por três ou mais dias na semana e durante 20 ou mais minutos por sessão, mais atividade moderada e/ou caminhada por cinco ou mais dias na semana e durante 30 ou mais minutos por sessão; Ativo, indivíduo que cumpriu as recomendações de atividade vigorosa por três ou mais dias na semana e durante 20 ou mais minutos por sessão. Ou atividade moderada e/ou caminhada por cinco ou mais dias na semana e durante 30 ou mais minutos por sessão. Ou qualquer atividade (vigorosa, moderada e/ ou caminhada) somada igual ou superior a cinco dias na semana durante 150 minutos ou mais por semana; Irregularmente ativo, indivíduo que realizou atividade física, contudo não atingiu os critérios supracitados. Pode ser classificado em: Irregularmente ativo $A$, praticou atividade física por, pelo menos, cinco dias na semana ou durante 150 minutos por semana; Irregularmente ativo $B$, não atingiu nem a frequência nem a duração recomendados; Sedentário, indivíduo que não praticou qualquer atividade física por, pelo menos, dez minutos contínuos durante a semana. Posteriormente, para análise estatística, os indivíduos foram categorizados em inativos (sedentário) e ativos (muito ativo, ativo e irregularmente ativo).

Para rastreamento de Transtorno Mental Comum (TMC), utilizou-se o instrumento Self-Reporting Questionnaire-20 (SRQ-20) ${ }^{16}$ que é constituído por 20 itens, com respostas dicotômicas ( im ou não), onde as respostas “sim” valem um ponto e as respostas "não" valem zero. Este questionário permite classificar o indivíduo quanto à presença ou ausência de potencial para TMC. A classificação é baseada no somatório das questões afirmativas. Indivíduos que apresentaram pontuação igual ou superior a sete foram classificados como "com potencial para TMC". Para fins de análise, esta variável foi dicotomizada em "sem potencial para TMC" e "com potencial para TMC".

O consumo alimentar foi avaliado por meio do Recordatório de 24 horas (R24h), que foi aplicado em um dia, podendo ser da semana ou final de semana. A aplicação do R24H seguiu o método dos múltiplos passos. Utilizou-se o software aplicado no Estudo de Riscos Cardiovasculares em Adolescentes - ERICA ${ }^{17}$ para a digitação dos dados. A base de dados do programa é a mesma que foi desenvolvida pelo Instituto Brasileiro de Geografia e Estatística (IBGE) em 2008/2009 na realização do Inquérito Nacional de Alimentação (INA). O consumo de nutrientes foi ajustado pelo método dos resíduos, proposto por Willett ${ }^{18}$. Para análise das variações do consumo alimentar na dieta dos universitários, levou-se em consideração as médias ajustadas para o consumo de energia, carboidratos, lipídios, proteínas, fibras, sódio e açúcar de adição.

As variáveis numéricas foram descritas por meio de média e desvio padrão e as categóricas por proporção (\%). Aplicou-se o teste de Kolgomorov-Smirnov para verificar a simetria das distribuições. Todas as distribuições assimétricas foram transformadas em logarítmos. O teste do Qui-quadrado foi utilizado para comparar os indivíduos com e sempotencial para TMC. A diferença entre as médias ajustadas de ingestão de energia e nutrientes foi testada por meio do Teste T de Student.

Desenvolveram-se modelos de regressão linear para avaliar a relação entre ingestão de energia e nutrientes e potencial para TMC. Os modelos foram desenvolvidos separadamente para cada nutriente (carboidratos, proteínas, gorduras totais, gordura saturada, fibras, açúcar de adição e sódio) e energia. Inicialmente ajustou-se por idade e sexo e em seguida por idade, sexo e atividade física. As análises foram realizadas no software SAS, versão online, considerando significância estatística quando p-valor $<0,05$.

O estudo foi aprovado pelo Comitê de Ética em Pesquisa da Universidade Estadual do Ceará. A participação na pesquisa foi condicionada à assinatura do Termo de Consentimento Livre e Esclarecido.

\section{Resultados}

Dos 432 universitários selecionados para participar do estudo, foram excluídos 30 participantes por não preencherem o questionário completamente. A análise dos dados foi então realizada com 402 estudantes universitários, com idade entre 18 e 60 anos (média da idade $=21,3$ anos, $\mathrm{DP}=3,2)$. Destes, $64,4 \%(\mathrm{n}=259)$ eram do sexo feminino, $66,4 \%(n=267)$ se autodeclararam de raça/cor da pele não branca, $11,9 \%$ apresentavam excesso de peso, 24,6\% foram classificados como ativos e 74,6\% apresentavam renda familiar menor ou igual a três salários mínimos.

A prevalência de rastreamento positivo para TMC foi de 44,5\%, sendo a mediana do SRQ20 igual a 6 pontos. Dentre os entrevistados, as mulheres apresentaram maior frequência de ras- 
treamento positivo para TMC quando comparadas aos homens $(53,3 \%$ vs. $28,7 \%, \mathrm{p}<0,001)$. O curso de enfermagem foi o que apresentou maior prevalência de rastreamento positivo para TMC (56,9\%), seguido do curso de Nutrição $(43,4 \%)$, Educação Física $(40,8 \%)$ e Ciências Biológicas (40\%). O curso de medicina apresentou menor percentual para o rastreamento positivo de TMC $(34,7 \%)$ (Tabela 1$)$.

Ao avaliarmos os comportamentos de estilo de vida e o potencial para TMC, observamos que as prevalências de inatividade física foram maiores entre os indivíduos classificados como com potencial para desenvolver TMC, quando comparados aos que não apresentaram potencial $(45,8 \%$ vs. $33,2 \%)$, sendo essa diferença estatisticamente significativa (Tabela 1).

A média de ingestão de açúcar de adição foi de 78,8 gramas por dia (g/dia) na população em geral. Os indivíduos que foram classificados como com potencial para TMC apresentaram, quando comparados aos sem potencial para desenvolver TMC, maior ingestão de açúcar de adição $(90,2 \mathrm{~g} /$ dia $v s .71,2 \mathrm{~g} /$ dia $)$ e gordura saturada $(29,7 \mathrm{~g} /$ dia $v s .27,5 \mathrm{~g} /$ dia $)$ e menor ingestão de fibras e sódio (15,1 g/dia vs. 17,3 g/dia; 1629,1 $\mathrm{mg} / \mathrm{dia}$ vs. 1853,6 mg/dia), sendo essas diferenças estatisticamente significativas. (Tabela 2).

Observou-se associação direta entre TMC e a ingestão de gorduras saturadas e açúcar de adição, no entanto após ajuste por idade, sexo e atividade física a significância estatística não se manteve para a ingestão de gorduras saturadas (Tabela 3). A relação entre TMC e ingestão de fibras e sódio foi negativa, independente da idade, sexo e da prática de atividade física.

\section{Discussão}

Observou-se no presente estudo, que quase metade da população estudada apresentou rastrea-

Tabela 1. Características sociodemográficas e comportamento de estilo de vida de estudantes de cursos da saúde de uma universidade pública, segundo rastreamento para transtorno mental comum. Fortaleza, Ceará, 2018.

\begin{tabular}{|c|c|c|c|c|}
\hline \multirow{3}{*}{ Variável } & \multicolumn{4}{|c|}{ Potencial para Transtorno } \\
\hline & \multirow[t]{2}{*}{ Total (n) } & \multicolumn{2}{|c|}{ Mental Comum } & \multirow[t]{2}{*}{ p-valor } \\
\hline & & Não (\%) & $\operatorname{Sim}(\%)$ & \\
\hline \multicolumn{5}{|l|}{ Sexo } \\
\hline Masculino & 143 & 45,7 & 22,9 & $\leq 0,01$ \\
\hline Feminino & 259 & 54,3 & 77,1 & \\
\hline \multicolumn{5}{|l|}{ Faixa etária } \\
\hline$\leq 21$ anos & 246 & 61,4 & 60,9 & 0,9 \\
\hline$>21$ anos & 156 & 38,6 & 39,1 & \\
\hline \multicolumn{5}{|l|}{ Curso } \\
\hline Ciências biológicas & 55 & 14,8 & 12,3 & 0,05 \\
\hline Educação física & 120 & 31,8 & 27,4 & \\
\hline Enfermagem & 102 & 19,7 & 32,4 & \\
\hline Medicina & 49 & 14,4 & 9,5 & \\
\hline Nutrição & 76 & 19,3 & 18,4 & \\
\hline Semestre & & & & 0,5 \\
\hline Início $\left(\leq 4^{\circ}\right)$ & 222 & 56,5 & 53,6 & \\
\hline Final $\left(\geq 5^{\circ}\right)$ & 180 & 43,5 & 46,4 & \\
\hline \multicolumn{5}{|l|}{ Renda familiar per capita } \\
\hline$\leq 3$ salários & 259 & 73,8 & 75,6 & 0,7 \\
\hline$>3$ salários & 88 & 26,2 & 24,4 & \\
\hline \multicolumn{5}{|l|}{ Raça/Cor da pele } \\
\hline Branca & 135 & 30,5 & 37,4 & 0,1 \\
\hline Outras & 267 & 69,5 & 62,6 & \\
\hline \multicolumn{5}{|l|}{ Estado civil } \\
\hline Solteiro & 382 & 96,0 & 93,9 & 0,8 \\
\hline Outros & 20 & 4,0 & 6,2 & \\
\hline \multicolumn{5}{|l|}{ Prática de atividade física } \\
\hline Inativo & 156 & 33,2 & 45,8 & $\leq 0,01$ \\
\hline Ativo & 246 & 66,8 & 54,2 & \\
\hline
\end{tabular}

Fonte: Elaborado pelos autores. 
Tabela 2. Média de ingestão de energia e nutrientes de estudantes de cursos da saúde de uma universidade pública, segundo rastreamento para transtorno mental comum. Fortaleza, Ceará, 2018.

\begin{tabular}{lrrrr}
\hline \multirow{2}{*}{ Energia ou Nutriente } & \multirow{3}{*}{ Total } & \multicolumn{2}{c}{ Potencial para Transtorno Mental } & \multirow{2}{*}{ P-valor } \\
\cline { 3 - 4 } & & \multicolumn{1}{c}{ Não } & \multicolumn{1}{c}{ Sim } & \\
\hline Energia (g/dia) & $2.311,3$ & $2.363,5$ & $2.231,2$ & 0,2 \\
Carboidratos (g/dia) & 272,1 & 273,3 & 271,9 & 0,8 \\
Açúcar de adição (g/dia) & 78,8 & 71,2 & 90,2 & $\leq 0,01$ \\
Fibras (g/dia) & 16,5 & 17,3 & 15,1 & $\leq 0,01$ \\
Gorduras Totais (g/dia) & 74,9 & 73,6 & 77,1 & 0,07 \\
Gorduras Saturadas (g/dia) & 28,1 & 27,5 & 29,7 & 0,03 \\
Proteínas (g/dia) & 92,0 & 93,1 & 88,2 & 0,1 \\
Sódio (mg/dia) & $1.742,5$ & $1.853,6$ & $1.629,1$ & 0,04 \\
\hline
\end{tabular}

${ }^{\star}$ Nutrientes ajustados pela ingestão total de energia.

Fonte: Elaborado pelos autores.

Tabela 3. Coeficiente de regressão linear $(\beta)$ da relação entre ingestão de energia e nutrientes e potencial para Transtorno Mental Comum em estudantes de cursos da saúde de uma universidade pública. Fortaleza, Ceará, 2018.

\begin{tabular}{lccc}
\hline \multicolumn{1}{c}{ Energia ou Nutriente } & Modelo bruto $(\boldsymbol{\beta})$ & $\begin{array}{c}\text { Modelo ajustado } \\
\text { por idade e sexo } \\
(\boldsymbol{\beta})\end{array}$ & $\begin{array}{c}\text { Modelo ajustado } \\
\text { por idade, sexo } \\
\text { e atividade física }(\boldsymbol{\beta})\end{array}$ \\
\hline Energia (kcal/dia) & $-132,3$ & 2,4 & $-16,0$ \\
Carboidratos (g/dia) & $-1,4$ & $-0,9$ & $-1,5$ \\
Açúcar de adição (g/dia) & $19,0^{*}$ & $16,2^{*}$ & $16,0^{*}$ \\
Fibras (g/dia) & $-2,2^{*}$ & $-1,9^{*}$ & $-1,9^{*}$ \\
Gorduras Totais (g/dia) & 3,5 & 2,7 & 2,6 \\
Gorduras Saturadas (g/dia) & $2,2^{*}$ & 1,7 & 1,7 \\
Proteínas (g/dia) & $-5,0$ & $-4,6$ & $-4,0$ \\
Sódio (mg/dia) & $-224,5$ & $-272,6^{*}$ & $-276,8^{*}$ \\
\hline
\end{tabular}

* p-valor $\leq 0,05$.

Fonte: Elaborado pelos autores.

mento positivo para TMC.Estudos realizados em outras regiões do país encontraram prevalências semelhantes às do presente estudo, os quais verificaram prevalências de TMCvariando de 31,5\% a $44,9 \%$ entre os universitários da área da saú$\mathrm{de}^{5,19} \mathrm{e} 40 \%$ nos estudantes de outras áreas ${ }^{6}$. Assim como no presente estudo, outras pesquisas realizadas no Brasil, com estudantes da área da saúde, também verificaram maior prevalência de TMC entre as mulheres ${ }^{20,21}$. Os fatores que poderiam explicar essa maior prevalência é o fato das mulheres apresentam diferenças sexuais ligadas a aspectos neuronais relacionados ao humor e a ansiedade e estarem expostas a estressores envolvidos com os aspectos psicossociais e as desigualdades associadas ao gênero ${ }^{22,23}$.
Os indivíduos com potencial para TMC apresentaram menor frequência de prática de atividade física. Consideremos que tal prática pode trazer efeito protetor quanto ao surgimento de sintomas relacionados ao $\mathrm{TMC}^{24}$. A prática regular de exercício físico provoca efeito positivo no estado de humor das pessoas, reduz a ansiedade e a depressão, enquanto a inatividade é tida como um fator de risco para o desenvolvimento de sintomas depressivos ${ }^{25,26}$.

Os indivíduos classificados como com potencial para TMC apresentaram maior ingestão de açúcar de adição, gordura saturada e menor ingestão de fibras e sódio. Verificou-se também, associação direta entre TMC e a ingestão de açúcar de adição. A ingestão de açúcar na popula- 
ção foi de $78,8 \mathrm{~g} / \mathrm{dia}$, o que equivale a $15,8 \%$ do valor calórico total de uma dieta de $2000 \mathrm{kcal}$, ultrapassando em $10 \%$ as recomendações de ingestão diária da Organização Mundial de Saúde $(\mathrm{OMS})^{27}$. Fernandes et al. ${ }^{9}$ encontraram resultados semelhantes ao verificarem, entre universitários da área da saúde, média de ingestão diária de açúcar de adição de 69,21g.

Pesquisa realizada com universitários da saúde, que investigou a constipação intestinal e fatores associados, constatou que $14,6 \%$ dos estudantes apresentavam quadros de constipação intestinal e quase metade dos voluntários referiu fazer a maioria das refeições fora do lar, optando por lanches prontos e fast foods, associando-se à densa carga horária e, principalmente, ao estresse do ambiente acadêmico, contribuindo para menor ingestão de fibras e outros nutrientes ${ }^{8}$.

A resposta biológica diante das situações de estresse produz distintas reações entre os indivíduos, entre elas, o aumento do apetite ${ }^{8,28}$. Essas condições podem alterar o comportamento alimentar, induzindo os indivíduos a comportamentos alimentares pouco saudáveis, como maior ingestão de fast-food, refeições prontas e lanches, pular refeições e comer demais ${ }^{29}$.

Estudo que avaliou o estado emocional e o comportamento alimentar de 54 universitárias constatou que as acadêmicas de nutrição e de farmácia consumiam doces quando se sentiam estressadas ( $23 \%$ e $43 \%$, respectivamente) e ansiosas $(27 \% \text { e } 43 \% \text {, respectivamente })^{30}$. Pesquisa realizada com 3.706 universitários do Reino Unido verificou associação positiva entre os níveis de estresse e consumo de snacks e fast food ${ }^{31}$. Por outro lado, estudo que avaliou o consumo alimentar em universitários classificados como de maior e menor nível de estresse, não verificou diferença no consumo de energia total e macronutrientes, no entanto, observaram maior frequência e regularidade de consumo de salgados, lanches prontos e fast food entre os estudantes categorizados como de maior carga de estresse ${ }^{32}$.

A literatura evidencia algumas situações tidas como desencadeadoras do estresse entre univer- sitários, dentre elas encontra-se deixar de morar com os pais, dividir moradia com novas pessoas, responder às próprias expectativas e às dos pais, dificuldade de organizar o tempo, privação do sono, gerir trabalho e/ou estudo em concomitante as atividades domésticas, a rotina intensa de estudo, estresse particular de cada curso e, por fim, preocupação em terminar o curso e a sua inserção no mercado de trabalho, ${ }^{33}$. Tais demandas tornam o universitário mais propenso a desequilíbrios emocionais como o estresse e a ansiedade, podendo levar a um insucesso acadêmico ${ }^{34}$.

Destaca-se, diante do exposto, a necessidade de atenção para as altas prevalências que os TMC vêm apresentando entre o público dos universitários, que, futuramente, serão profissionais de saúde. Nesse sentido, é essencial que esses profissionais estejam em boas condições mentais para, assim, assumir o papel de cuidador ou agente promotor de saúde de forma que suas ações possam apresentar eficiência e eficácia ${ }^{3,35}$.

Entre as limitações do presente estudo está o delineamento transversal que impossibilita estabelecer relações de causa e efeito e o fato do instrumento utilizado estar sujeito a viés na categorização, uma vez que há uma variedade de pontos de cortes adotados para classificação do rastreamento positivo. Entretanto, adotou-se a recomendação de Mari e Williams ${ }^{16}$, sendo a versão brasileira do instrumento, que adota o ponto de corte de sete perguntas afirmativas para considerar rastreamento positivo para TMC, a fim de corrigir possíveis erros.

\section{Conclusão}

A prevalência de universitários com potencial para TMC entre o público universitário foi elevada e apresentou associação com o sexo feminino, com inatividade física e com maior ingestão de açúcar de adição, gorduras saturadas e menor ingestão de fibras. 


\section{Colaboradores}

AR Sousa trabalhou na concepção e delineamento da pesquisa, na coleta, digitação, análise, interpretação dos dados e na redação do texto. DM Reis trabalhou na concepção e delineamento da pesquisa, na coleta, digitação, análise, interpretação dos dados e na redação do texto. TM Vasconcelos interpretação dos dados e da redação e revisão final do manuscrito. APV Abdon coordenou a pesquisa, participaram da análise e interpretação dos dados e da redação e revisão final do manuscrito. SP Machado coordenou a pesquisa e participou da análise e interpretação dos dados e revisão final do manuscrito. IN Bezerra coordenou a pesquisa, participou da análise e interpretação dos dados e da redação e revisão final do manuscrito.

\section{Referências}

1. Auerbach RP, Alonso J, Cuijpers P, Ebert DD, Hasking P, Nock MK, Stein DJ, Kessler RC, Zaslavsky AM, Mortier P, Bruffaerts R, Benjet C, Demyttenaere K, Gree JG, Murray E, Pinder-Amaker S, Sampson NA, Vilagut G. WHO World Mental Health Surveys International College Student Project: Prevalence and distribution of mental disorders. J Abnorm Psychol 2018; 127(7):623-638.

2. Goldberg D, Huxley P. Common mental disorders: a bio-social model. 1a Edição. London: Tavistock/Routledge; 1992.

3. Ansolin AGA, Rocha DLB, Santos RP, Pozzo VCD. Prevalência de transtorno mental comum entre estudantes de psicologia e enfermagem. Arqui Cien Saude 2015; 22(3):42-45.

4. Haile YG, Alemu SM, Habtewold TD. Common mental disorder and its association with academic performance among Debre Berhan University students, Ethiopia. Int J Ment Health Syst 2017; 11(34):02-11.

5. Pacheco JP, Giacomin HT, Tam WW, Ribeiro TB, Arab C, Bezerra IM, Pinasco Gustavo C. Mental health problems among medical students in Brazil: a systematic review and meta-analysis. Braz J Psychiatry 2017; 39(4):369-378

6. Silva AO, Neto JLC. Associação entre níveis de atividade física e transtorno mental comum em estudantes universitários. Motri 2014; 10(1):49-59.

7. Perini JP, Delanogare E, Souza SA. Transtornos mentais comuns e aspectos psicossociais em universitários do sul do Brasil. Vittalle-RevCicenSaude 2019; 31(1):44-51.

8. Deliens T, Clarys P, Bourdeaudhuij I, Deforche B. Determinants of eating behaviour in university students: A qualitative study using focus group discussions. BMC Public Health 2014; 14(53):1-12.

9. Fernandes DPS, Cândido FG, Rocha JLM, Reis VG, Souza ECG, Duarte MSL. Ingestão de açúcar de adição por estudantes da área da saúde em uma instituição de ensino de Viçosa, Brasil. Mundo Saude 2016; 40(1):94-105.

10. Silva DAS, Petroski EL. The simultaneous presence of health risk behaviors in freshman college students in Brazil. J Community Health 2012; 37(3):591-598.

11. Deasy C, Coughlan B, Pironom J, Jourdan D, Mannix-Mcnamara P. Psychological Distress and Coping amongst Higher Education Students: A Mixed Method Enquiry. PloS One 2014; 9(12):1-23.

12. Cochran WG. Sampling Techniques. 3. Edição. Nova York: John Wiley; 1977.

13. Instituto Brasileiro de Geografia e Estatística (IBGE). Pesquisa de OrçamentosFamiliares - POF 2008 - 2010. Antropometria e Estado Nutricional de Crianças, Adolescentes e Adultos no Brasil. Rio de Janeiro: IBGE; 2010.

14. Matsudo S, Araújo T, Marsudo V, Andrade D, Andrade E, Oliveira LC, Braggion G. Questionário Internacional de Atividade Física (IPAQ): Estudo de validade e reprodutibilidade no Brasil. RevBrasAtivFís Saúde 2001; 6(2):5-18.

15. Centro Coordenador do IPAQ no Brasil. Classificação e avaliação do nível de atividade física, 2007. [acessado 19 fev 2020]. Disponível em: https://celafiscs.org.br/ articles/. 
16. Mari JJ, Williams P. A validity study of a psychiatric screening questionnaire (SRQ-20) in primary care in the city of Sao Paulo. J Bras Psiquiatr 1986; 22(2):6-23.

17. Barufaldi LA, Abreu GA, Veiga GV, Sichieri R, Kuschnir MCC, Cunha DB, Pereira RA, Bloch KV. Programa para registro de recordatório alimentar de 24 horas: aplicação no Estudo de Riscos Cardiovasculares em Adolescentes. Rev Bras Epidemiol 2016; 19(2):464468.

18. Willett WC, Howe GR, Kushi LH. Adjustment for total energy intake in epidemiologic studies. Am J Clin Nutr 1997; 65(4 Supl.):1220S-1231S.

19. Graner KM, Moraes ABAd, Torres AR, Lima MCP, Rolim GS, Ramos-Cerqueira ATA. Prevalence and correlates of common mental disorders among dental students in Brazil. PLoS ONE 2018; 13(9):e0204558.

20. Silva PLBC, Silva BFF, Chagas KKAR, Tortola MBA, Caldeira RLR. Transtorno mental comum entre estudantes de enfermagem e fatores envolvidos. Ver Enferm Cent-Oeste Min 2019; 9:3.191-3.197.

21. Sakae TM, Padão DL, Jordana LK. Sintomas depressivos em estudantes da área da saúde em uma universidade no sul de Santa Catarina - UNISUL. Rev AMRIGS 2010; 54(1):38-43.

22. Patel V, Araya R, Lima M, Ludermir A, Todd C. Women, poverty and common mental disorders in four restructuring societies. Soc Sci Med 1999; 49(11):1.461-1.471.

23. Joel D, Berman Z, Tavor I, Wexler N, Gaber O, Stein Y, Shefi N, Pool J, Urchs S, Margulies DS, Liem F, Hänggi J, Jäncke L, Assaf Y. Sex beyond the genitalia: the human brain mosaic. PNAS 2015; 112(50):15.46815.473.

24. Harvey SB, Hotopf M, Øverland S, Mykletun A. Physical activity and common mental disorders. British $J$ Psychiat 2010; 197(5):357-364.

25. Rimer J, Dwan K, Lawlor DA, Carolyn AG, Marion MM, Wendy M, Gillian EM. Exercise for depression. Cochrane Database Syst Rev 2012; (7):CD004366.

26. Schuch FB, Vancampfort D, Richards J, Rosenbaum S, Ward PB, Stubbs B. Exercise as a treatment for depression: A meta-analysis adjusting for publication bias. $J$ Psychiatr Res 2016; 77:42-51.

27. Organização Mundial de Saúde (OMS). Ingestão de açúcares por adultos e crianças. Genebra: OMS; 2015. [acessado 2020 fev 19]. Disponível em: https://www. paho.org/bra/images/stories/GCC/ingestao $\% 20$ de $\% 20$ acucares $\% 20$ por\%20adultos\%20e\%20criancas_portugues.pdf?ua $=1$.
28. Wallis DJ, Hetherington MM. Emotions and eating. Self-reported and experimentally induced changes in food intake under stress. Appetite 2009; 52(2):355362.

29. Choi J. Impact of Stress Levels on Eating Behaviors among College Students. Nutrients 2020; 12(5):1.241

30. Pereira TC, Sousa FGA, Moreira AS, Santos JL, Patrício AMD, Bueno MM. Estado emocional e comportamento alimentar de universitárias de uma instituição de ensino particular. Rev E-ciência 2019; 7(1):16-20.

31. El Ansari W, Adetunji H, Oskrochi R. Food and mental health: relationship between food and perceived stress and depressive symptoms among university students in the United Kingdom. Cent Eur J Public Health 2014; 22(2):90-97.

32. Penaforte FRO, Matta NC, Japur CC. Associação entre estresse e comportamento alimentar em estudantes universitários. Demetra 2016; 11(1):225-237.

33. Vasconcelos-Raposo J, Soares AR, Fernandes MG, Teixeira CM. Níveis de ideação suicida em jovens adultos. Estud psicol 2016; 22(2):345-354.

34. El Ansari W, Berg-Beckhoff G. Nutritional correlates of perceived stress among university students in Egypt. Int J Environ Res Public Health 2015; 12(11):14.164-14.176.

35. Araújo AHI. Influências de quadros de ansiedade e depressão no consumo alimentar em adultos jovens saudáveis [dissertação]. Brasília: Universidade de Brasília; 2017.

Artigo apresentado em 23/03/2020

Aprovado em 26/06/2020

Versão final apresentada em 28/06/2020

Editores-chefes: Romeu Gomes, Antônio Augusto Moura da Silva 